\title{
Heterogeneous Spectrum Sensing in Cognitive Radio Network using Traditional Energy Detection and Matched Filter
}

\author{
Shahbaz Soofi \\ Department of Electrical \& \\ Electronics Engineering, NITTTR \\ Bhopal, India
}

\author{
Anjali Potnis \\ Department of Electrical \& \\ Electronics Engineering, NITTTR \\ Bhopal, India
}

\author{
Prashant Dwivedy \\ Department of Electrical \& \\ Electronics Engineering, NITTTR \\ Bhopal, India
}

\begin{abstract}
The accurate spectrum sensing is a predominant aspect of any competent CR system. Efficient spectrum sensing enables a CR terminal to detect the spectrum holes (underutilized spectral bands) by providing high spectral resolution, thereby accrediting opportunistic transmission in the licensed band to the CR. In order to facilitate a good spectrum management and its efficient use a hybrid method for the detection of the spectrum with the purpose of detecting the presence of bands of unoccupied frequencies is proposed. The method used are traditional energy detection and matched filter with changing number of secondary users using each technique and finally a centralized cooperative spectrum sensing network which employs hard combination at the fusion centre.
\end{abstract}

Keywords- cognitive radio; spectrum sensing; energy detection, ROC; Matched Filter

\section{INTRODUCTION}

Given the limitations of the natural frequency spectrum, it becomes obvious that the current static frequency allocation schemes cannot accommodate the requirements of an increasing number of higher data rate devices. As a result, innovative techniques that can offer new ways of exploiting the available spectrum are needed. Cognitive radio arises to be a tempting solution to the spectral congestion problem by introducing opportunistic usage of the frequency bands that are not heavily occupied by licensed users. In effect, this approach proposes to a new category of users so-called secondary users (SUs) to access the frequency resources allocated to primary users (PUs) when the latter do not use them [1]. Primary users can be defined as the users who have higher priority or legacy rights on the usage of a specific part of the spectrum. On the other hand, secondary users, which have lower priority, exploit this spectrum in such a way that they do not cause interference to primary users. Therefore, secondary users need to have cognitive radio capabilities, such as sensing the spectrum reliably to check whether it is being used by a primary user and to change the radio parameters to exploit the unused part of the spectrum. Thus, the spectral efficiency is increased by allowing the transmission by the SUs on frequency bands detected free .

Being the focus of this paper, spectrum sensing by far is the most important component for the establishment of cognitive radio. Spectrum sensing is the task of obtaining awareness about the spectrum usage and existence of primary users in a geographical area. The detection of the spectrum is hence a crucial task for the success of the opportunistic access. It is a cardinal feature of cognitive radio to avoid harmful interference with authorized users and recognize the spectrum available to improve the use of the spectrum.

There are several spectrum detection techniques. In the scope of this paper we have evaluated the results of spectrum detection using traditional energy detection and then compared the result with proposed hybrid sensing wherein a certain percentage of the nodes where using traditional energy detection and the remaining were designed to use matched filter detection for different number of secondary users.

Finally concept of cooperative spectrum sensing for cognitive radio networks is implemented to combat difficulties such as multipath fading, shadowing and receiver uncertainty issues. Here the information collected by each node is reported in the form of single or multi bit decisions called hard combination to the fusion centre which uses a majority rule to form final decision on the presence of primary user PU.

\section{Mathematical Modeling For Spectrum Sensing}

Spectrum sensing [2] is based on the signal detection theory and a sensing device can be viewed as a binary classifier making a decision out of a two hypothesis test where the hypothesis $\mathrm{H} 0$ and $\mathrm{H} 1$ represent the absence and presence of a PU respectively.

$$
y(k)=\left\{\begin{array}{lll}
n(k) & : & H 0 \\
n(k)+p(k) & : & H 1
\end{array}\right.
$$

Here $y(k)$ represents the detected signal, $n(k)$ represents noise and $\mathrm{p} \mathrm{(k)}$ refers to the PU signal. Four possibilities exist for the detection:

Declaring $\mathrm{H} 0$ when $\mathrm{H} 0$ is true $(\mathrm{H} 0 \mid \mathrm{H} 0)$ : Correct Detection.

Declaring $\mathrm{H} 1$ when $\mathrm{H} 1$ is true $(\mathrm{H} 1 \mid \mathrm{H} 1)$ : Correct Rejection.

Declaration $\mathrm{H} 0$ when $\mathrm{H} 1$ is true $(\mathrm{H} 0 \mid \mathrm{H} 1)$ : False Alarm.

Declaring $\mathrm{H} 1$ when $\mathrm{H} 0$ is true (H1|H0): Missed Detection.

Thus, correct detections are the true positives, correct rejections are the true negatives, false alarms are the false 
positives and missed detections are the false negatives for the spectrum sensing test [3].

\section{SPECTRUM SENSING METHODS FOR COGNITIVE RADIO}

A number of different methods are proposed for identifying the presence of signal transmissions. In this section, some of the most common spectrum sensing techniques available in the cognitive radio literature is explained.

\section{A. Energy Detector Based Sensing}

Energy detector based approach, also known as radiometry or period gram, is the most common way of spectrum sensing because of its low computational and implementation complexities. In addition, it is more generic (as compared to methods given in this section) as receivers do not need any knowledge on the primary users' signal. . The traditional Energy Detector [4] simply measures the energy of the received signal over a fixed interval termed as sensing interval. The average energy calculated over the sensing interval becomes the test statistic. The test statistic is then compared with a pre defined threshold. If the value of the test statistic is greater than the value of the threshold, the frequency being sensed is declared to be occupied otherwise it is declared as free to be used by the SU.

The design and performance parameters of traditional energy detector are the characteristics that are input to its design process. An energy detector is designed for a desired false alarm probability. The target probability of false alarm decides the threshold employed in the decision logic. Thus for any energy detector the false alarm probability and decision threshold are predefined. The performance parameters include the probability of detection at the given desired probability of false alarm.

\section{- Design parameters}

An energy detector is designed for a predefined value of false alarm probability $(\mathrm{Pf})$ which also sets the decision threshold .For a target probability of false alarm Pf, the threshold and probability of detection and be expressed in terms of the inverse $Q$ function of the target false alarm probability given by equation

$$
\lambda=\sigma^{2}\left[\sqrt{\frac{2}{N}} Q^{-1}(\mathrm{Pf})+1\right]
$$

Here Pd, Pf, $\lambda$ represent the probability of false alarm, probability of detection and decision threshold for traditional energy detection respectively. Q represents the $\mathrm{Q}$ tail function, $\mathrm{N}$ and $\gamma$ represents the length of sensing interval and SNR observed at the node.

\section{- $\quad$ Performance Parameters}

For any energy detector designed for a particular target frequency and employing a given threshold, probability of detection is given in terms of the $\mathrm{Q}$ function (tail probability function of the normal distribution)

$$
P d=Q\left\{\frac{\left[\left(\frac{\lambda}{\sigma^{2}}\right)-\gamma-1\right] \sqrt{\frac{N}{2}}}{\gamma+1}\right\}
$$

Here Pd, Pf, $\lambda$ represent the probability of false alarm, probability of detection and decision threshold for traditional energy detection respectively. Q represents the $\mathrm{Q}$ tail function, $\mathrm{N}$ and $\gamma$ represent the length of sensing interval and SNR observed at the node.

Some of the challenges with energy detector based sensing include selection of the threshold for detecting primary users, inability to differentiate interference from primary users and noise, and poor performance under low signal-to-noise ratio (SNR) values. Moreover, energy detectors do not work efficiently for detecting spread spectrum signals.

\section{B. Matched Filter Detection}

Matched Filter Spectrum Detection: If SUs know the information about a PU signal [5] a priori, in this case, the optimal detection method is the matched filter since it maximizes the SNR of the received signal. Detection MF needs just a little time in order to obtain a good performance unlike other methods, such as the low probability of false alarm and missed detection, since the MF has less need for the samples received. As disadvantages, MF has an implementation complexity and high power consumption, because of the need of the detector receivers for all types of signals and the algorithms of corresponding receiver to be executed. The process screening requires a perfect knowledge of the PU signal and its frequency of operation, for example, the modulation type and order, the bandwidth, the pulse shape, packet format etc [6]. Whenever the information used by the matched filter is false the efficiency of the detection will be degraded, which leads to distortion of the CR concept and from the PU's perspective, may cause low QoS for the licensed users.

The matched filter is a system of linear filter used in the framework of the digital signal processing. It is used to optimize the SNR in presence of the additive noise stochastic. It provides the coherent detection. A signal received from primary user is transmitted through AWGN (Additive White Gaussian Noise) channel and the transmitted signal is applied to matched filter. Matched filter correlates the signal with time modified version and comparison between the predetermined threshold and the final output of matched filter will determine the presence of the primary user.

If $\mathrm{y}(\mathrm{k})$ is sequence of received samples at instant $\mathrm{k} \in\{1,2$. $\ldots \mathrm{N}\}$ at the signal detector, the decision rule can be stated as

$$
\text { Decide for } \begin{cases}H 0, & \text { if } \hat{S}<\gamma \\ H 1, & \text { if } \hat{S}>\gamma\end{cases}
$$


Where $\hat{S}=\sum_{k=1}^{N} y(k) x(k)^{*}$ is the decision criterion, $\gamma$ is the threshold to be compared and $x(k)^{*}$ is the transpose conjugate of the input sequence [7][8].

$\hat{S}$ is Gaussian

$$
\hat{S}= \begin{cases}\mathrm{N}\left(0, \sigma_{n}^{2} \varepsilon\right), & H_{0} \\ \mathrm{~N}\left(\varepsilon, \sigma_{n}^{2} \varepsilon\right), & H_{1}\end{cases}
$$

Where $\sigma_{n}^{2}$ is the variance of the noise and $\varepsilon$ is considered as the Energy of the signal [9] given by

$$
\varepsilon=\sum_{k=1}^{N} x(k)^{2}
$$

Based on this, the probabilities of false alarm Pf and detection Pd are:

$$
P_{f}=Q\left(\frac{\hat{s}}{\sqrt{\varepsilon \sigma_{n}^{2}}}\right)
$$

and

$$
P_{d}=Q\left(\frac{\hat{s}-\varepsilon}{\sqrt{\varepsilon \sigma_{n}^{2}}}\right)
$$

\section{HARD COMBINATION For COPERATIVE SENSING}

In this scheme, each cognitive radio (CR) user makes a decision on the state (presence or absence) of the PU and sends it in one bit form to the fusion center (FC). The main advantage of this method [10] is the easiness the fact that it needs limited bandwidth. Three rules for Hard Combination are described in the literature, these are AND Rule, OR rule and Majority rule. It was found that the Majority rule gave the best results and has been discussed below

$\mathrm{K}$ out of $\mathrm{N}$ or MAJORITY rule: Decides on the signal presence if at least $\mathrm{K}$ of the $\mathrm{N}$ users detected a signal with $1 \leq$ $\mathrm{K} \leq \mathrm{N}$.

The $\mathrm{K}$ out of $\mathrm{N}$ rule is also known as Half Voting rule when $\mathrm{K}$ is greater than or equal to $\mathrm{N} / 2$ i.e. a $\mathrm{PU}$ is declared to be present only if half or more than half of the total CR users detect it. The K- out of- $\mathrm{N}$ rule boils down to the AND rule for $\mathrm{K}=\mathrm{N}$ and to the $\mathrm{OR}$ rule for $\mathrm{K}=\mathrm{N}$. Thus, the $\mathrm{K}$ out of $\mathrm{N}$ rule [11][12] is the generalized form with AND and OR rules representing the two extremes.

\section{Proposed Model For Heterogenous Co- OPERATIVE SENSING}

As traditional energy detector fails in low SNR scenarios [13]. The improved detection algorithm efficiently manages to overcome these drawbacks by replacing some of the traditional energy detection nodes with matched filter detection. Hence instead of all the nodes carrying out spectrum sensing using energy detection a certain percentage detect the PU using matched filter technique [14]. To keep the overhead less all the nodes are not made to use matched filter and also matched filter requires coherent reception, which is generally hard to achieve in practice. So as to make a foolproof sensing process a balance is made between the adopted methodologies. Fig 1 describes the proposed block diagram

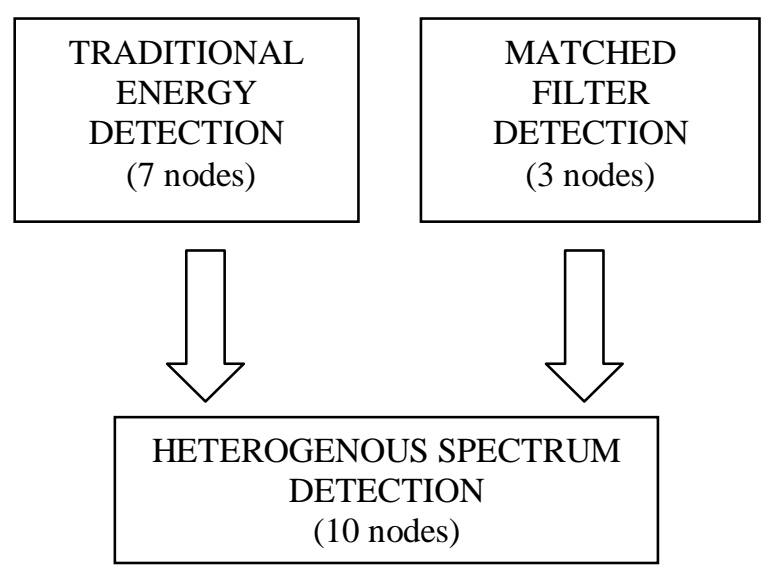

Figure 1. Proposed block diagram

\section{Simulation}

As mentioned above, the matched filter detector performance is based on two parameters $\mathrm{P}_{\mathrm{d}}$ andP $\mathrm{P}_{\mathrm{f}}$. Performance is shown by plotting curve between the two called the Receiver Operating Characteristic (ROC) curve [15]. The simulation was focused on AWGN Channel. Each point of the curve corresponds to a value of the set (PD, PFA) for a given threshold. When threshold increases, $P_{d}$ and $P_{f}$ decrease and, when threshold decreases, $P_{d}$ and $P_{f}$ increase [16].

We consider a heterogeneous cooperative spectrum sensing for a centralized network consisting of a cognitive base station (fusion centre) and a number of nodes collaborating in a non fading (AWGN) environment [17] with perfect reporting and sensing channels. The network assumed employs the optimized energy detection described in section II, at every node, for local sensing and adopts hard combination for information fusion at the fusion centre. The simulations are done in MATLAB version $\mathrm{R} 2013 \mathrm{a}$ for $\mathrm{SNR}=-14 \mathrm{db}$, the number of received signal samples $\mathrm{L}=100$ [18] and for three sets of SU nodes such that the ratio of number of nodes operating on traditional ED to the number of nodes operating on MF is always 2.33 for optimal results.

In the first run 7 nodes are made to run on traditional energy detection and 3 nodes on matched filter. The obtained results are compared with 10 nodes running on traditional energy detection and the obtained ROC is plotted in Fig 2. 


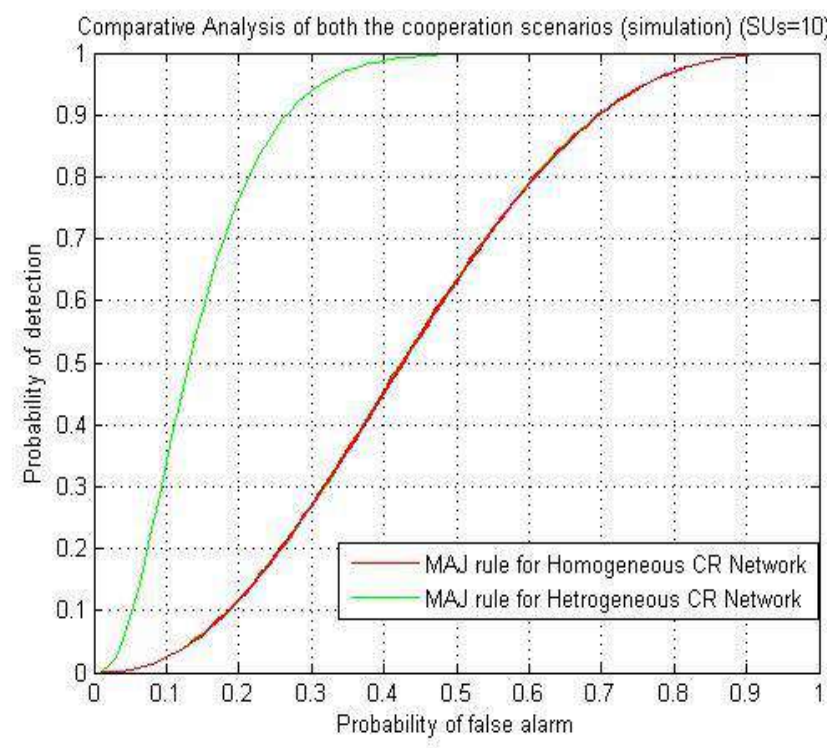

Figure 2. ROC curve for 7 traditional ED and $3 \mathrm{MF}$ v/s 10 traditional ED

In the next run, 14 nodes are made to run on traditional energy detection and 6 nodes on matched filter. The obtained results are compared with 20 nodes running on traditional energy detection and the obtained ROC is plotted in Fig 3.

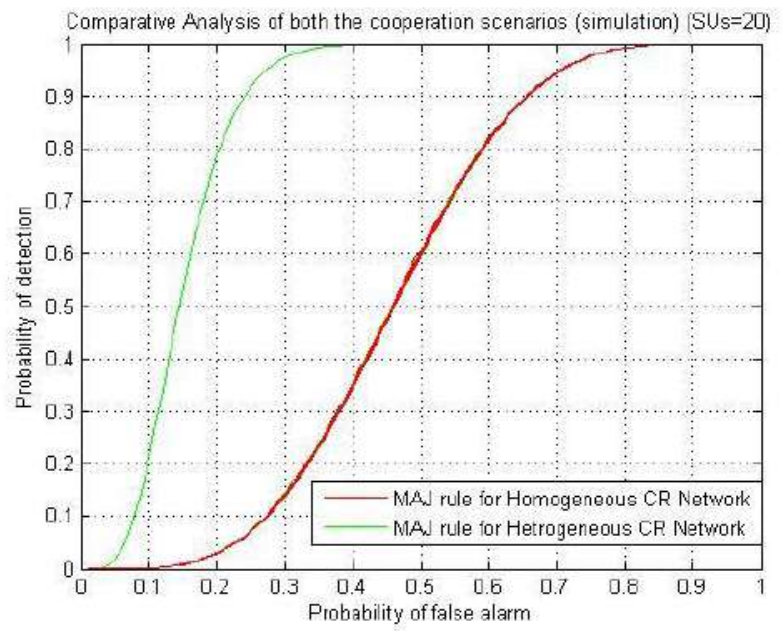

Figure 3. ROC curve for 14 traditional ED and $6 \mathrm{MF}$ v/s 20 traditional ED

In the next run 35 nodes are made to run on traditional energy detection and 15 nodes on matched filter. The obtained results are compared with 50 nodes running on traditional energy detection and the obtained ROC is plotted in Fig 4.

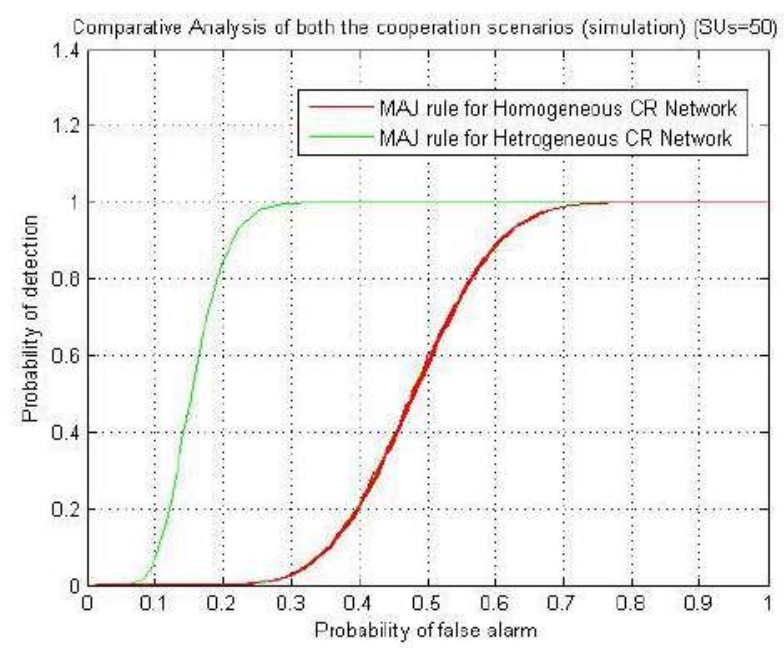

Figure 4. ROC curve for 35 traditional ED and $15 \mathrm{MF}$ v/s 50 traditional ED

\section{CONCLUSION}

In this paper, the technique for detection of the spectrum using a heterogeneous model employing the use of energy detection and matched filter is presented. The result obtained through the curves, shows that this method of detection maximizes the SNR of the received and probability of detection compared to the other homogenous methods.

In future traditional energy detection can be replaced by enhanced energy detector and other spectrum detection techniques can be explored.

\section{REFERENCES}

[1] G. Ziafat S, Ejaz W, Jamal H. Spectrum sensing techniques for cognitive radio networks:Performance analysis// Intelligent Radio for Future Personal Terminals (IMWS-IRFPT), 2011 IEEE MTT-S International Microwave Workshop Series onIEEE, 2011:1-4.

[2] Kalambe S, Lohiya P, Malathi P. Performance evolution of energy detection spectrum sensing technique used in cognitive radio// Signal Propagation and Computer Technology (ICSPCT), 2014 International Conference onIEEE, 2014:786-790.

[3] Digham FF, Alouini M-S, Simon MK. On the Energy Detection of Unknown Signals Over Fading Channels. Communications, IEEE Transactions on. 2007;55(1):21-4.

[4] Urkowitz H. Energy detection of unknown deterministic signals. Proceedings of the IEEE. 1967;55(4):523-531.

[5] Letaief KB, Zhang W. Cooperative Communications for Cognitive Radio Networks. Proceedings of the IEEE. 2009;97(5):878-93.

[6] Sahai A, Hoven N, Tandra R. Some fundamental limits on cognitive radio. Allerton Conference on Control, Communications, and Computation. 2004. p. 1662-71.

[7] J.Song, Z.Feng, P.Zhang, Z.Liu, "Spectrum sensing in cognitive radios based on enhanced energy detector," IET Communications, Vol.6, No.8, pp.805-809, May 2012.

[8] W.Zhang, R.K.Mallik, K.B.Letaief, "Optimization of Cooperative Spectrum Sensing with Energy Detection in Cognitive Radio Networks, IEEE Transactions in Wireless Communications, Vol.8, No.12, pp. 5761 - 5766, December 2009.

[9] Y.Chang Liang, Y.Zeng, E.C.Y. Peh, A.T.Hoang, "Sensing-Throughput Tradeoff for Cognitive Radio Networks," IEEE Transactions on Wireless Communications, Vol.7, No.4, pp.1326-1337, April 2008. 
[10] J.Liza, K.Muthumeenakshi, S.Radha, "Cooperative Spectrum Sensing in a Realistic Cognitive Radio Environment", IEEE International Conference on Recent Trends in Information Technology, Chennai, pp 375 - 379, June 2011

[11] S.Chaudhari, J.Lunden, V.Koivunen, H.V.Poor., "Cooperative Sensing with Imperfect Reporting Channels: Hard decisions or Soft decisions?" IEEE transactions on signal processing, Vol.60, No.1, pp. 18-28, Jan 2012.

[12] H.Urkowitz, "Energy detection of unknown deterministic signals," in Proceedings of the IEEE, Vol. 55, No. 4, pp. 523 - 531, April 1967.

[13] M.L pez- en tez, F.Casadevall, "Improved energy detection spectrum sensing for cognitive radio," IET Communications, Vol.6, No.8, pp.785796, May 2012.

[14] A.K.Dey, A. anerjee, "On Primary User Detection using energy detection technique for cognitive radio", National Conference on Communications, IIT Guwahati, pp. 99 - 102, 2009.

[15] F.Digham, M.S. Alouini, M. K. Simon, "On the energy detection of unknown signals over fading channels," IEEE International Conference on Communications (ICC'03), Anchorage, AK, USA, pp. 3575-3579, May 2003.
[16] B.Alexander, R.D.Koilpillai, "Cognitive Radio Techniques for GSM and" National Conference on Communications, IIT Bombay, pp. $1-5$, 2008.

[17] Z.Ejaz, N.U.Hasan, M.A.Azam, H.S.Kim, "Improved local spectrum sensing for cognitive radio networks", Eurasip Journal of Advanced Signal Processing, Vol. 2012, pp. 1 - 12, 2012.

[18] Z.Ejaz, N.U. Hasan, H.S.Kim, "SNR ased adaptive spectrum sensing for cognitive radio networks", International Journal of Innovative Computing Information and Control, Vol.8, No.9, pp. 6095-6106 2012. 